\title{
Actividad antioxidante de hongos silvestres consumidos tradicionalmente en el centro de México
}

\section{Antioxidant activity of wild mushrooms traditionally consumed in central Mexico}

\author{
Azucena González-Morales 1,2, Rosa María Ribas-Aparicio 1, Cristina Burrola-Aguilar ${ }^{2}$ \\ ${ }^{1}$ Departamento de Microbiología, Escuela Nacional de Ciencias Biológicas, Instituto Politécnico Nacional. Prolongación de Carpio y \\ Plan de Ayala s/n, Santo Tomás, C.P. 11340, Alcaldía Miguel Hidalgo, Ciudad de México, México. \\ ${ }^{2}$ Centro de Investigación en Recursos Bióticos, Universidad Autónoma del Estado de México. Carretera Toluca-lxtlahuaca km 14.5, \\ San Cayetano. C. P. 50295, Toluca, Estado de México, México.
}

\section{RESUMEN}

Antecedentes: Los hongos comestibles silvestres representan una valiosa fuente de alimento por su alto contenido de proteínas, vitaminas, fibra y bajos niveles de grasa. Además presentan compuestos bioactivos que incluyen compuestos fenólicos relacionados con propiedades antioxidantes.

Objetivos: Determinar la composición química, cuantificar el contenido de fenoles totales y evaluar la actividad antioxidante de seis especies de hongos comestibles silvestres consumidos tradicionalmente en el centro de México.

Métodos: Se obtuvieron extractos con agua, metanol, hexano y acetato de etilo a partir de los cuales se realizó un ensayo preliminar micoquímico. Posteriormente, se cuantificó el contenido de compuestos fenólicos totales por el método de Folin-Ciocalteu y finalmente, se evaluó la actividad antioxidante frente al radical DPPH.

Resultados y conclusiones: En los extractos fúngicos (Amanita rubescens, Flammulina mexicana, Floccularia aff. luteovirens, Gyromitra sp., Morchella sp., Turbinellus floccosus) se detectaron flavonoides, saponinas, taninos, quinonas, cumarinas y azucares reductores. En extractos acuosos se encontró la mayor concentración de compuestos fenólicos totales y actividad antioxidante, mientras que en extractos con acetato de etilo la menor. Los datos aquí obtenidos son el primer reporte para las especies de estudio, contribuyendo al conocimiento de la composición química y la actividad biológica de los hongos silvestres de México.

Palabras clave: extractos, compuestos fenólicos, fuente de alimento

\section{ABSTRACT}

Background: Wild edible mushrooms represent a valuable food source due to their high content of protein, vitamins, fiber, and low levels of fat. In addition many wild edible mushrooms have bioactive compounds that include phenolic compounds related to antioxidant properties.

Objective: To determine the chemical composition, quantify the content of total phenols and evaluate the antioxidant activity of six species of wild edible mushrooms traditionally consumed in Central Mexico.

Methods: Extracts with water, methanol, hexane and ethyl acetate were obtained from which a preliminary mycochemical test was carried out. Afterwards, the content of total phenolic compounds was quantified by the Folin-Ciocalteu method and finally, the antioxidant activity against the DPPH radical was evaluated.

Results and conclusions: In the different fungal extracts (Amanita rubescens, Flammulina mexicana, Floccularia aff. luteovirens, Gyromitra sp., Morchella sp., Turbinellus floccosus) flavonoids, saponins, tannins, quinones, coumarins and reducing sugars were detected. In aqueous extracts the highest concentration of total phenolic compounds and antioxidant activity was found, while extracts ethyl acetate the lowest. The data obtained is the first report for the study species, contributing to the knowledge of the chemical composition and biological activity of wild mushrooms in Mexico.

Keywords: extracts, phenolic compounds, food source

\section{ARTICLE HISTORY}

Received: 04 August 2021 / Accepted: 30 September 2021

Published on line: 18 November 2021

\section{CORRESPONDING AUTHOR}

Cristina Burrola-Aguilar, cba@uaemex.mx

ORCID 0000-0003-3499-8668 


\section{INTRODUCCIÓN}

Desde tiempos remotos, la humanidad ha considerado a los hongos silvestres una valiosa fuente de alimento y medicina. Antiguos pobladores reconocían las propiedades nutricionales y funcionales de los hongos, mismas que han sido comprobadas por diversas investigaciones científicas que han demostrado que su composición nutricional consiste en un alto contenido de proteínas, vitaminas, fibra y bajos niveles de grasa (Chaturvedi et al. 2018). Adicional a su valor nutrimental, muchos hongos silvestres presentan compuestos biológicamente activos tales como los compuestos fenólicos con propiedades antioxidantes, antimicrobianas, antivirales, anticancerosas, entre otras (Golak-Siwulska et al. 2018; Gebreyohannes et al. 2019). También se sabe que la composición química de los hongos varia de una especie a otra, así como del sustrato donde crecen, de su estadio de desarrollo, temperatura, humedad, entre otras variables geográficas y ambientales e incluso de la forma en que se preparan para su consumo (Guillamón et al. 2010; Sánchez 2017). Se estima que en el mundo hay 2300 especies de hongos silvestres comestibles y medicinales (Islam et al. 2019; Martínez-Medina et al. 2021). En México 450 especies son consumidas (Pérez-Moreno et al. 2020) y 350 son usadas en la medicina tradicional de los diferentes grupos originarios que habitan el país, quienes las utilizan para el tratamiento de más de 150 enfermedades (Bautista-González y Herrera-Campos 2019).

De entre toda la gama de compuestos bioactivos que producen los hongos se destacan los fenoles, reconocidos por su remarcada capacidad antioxidante (Barros et al. 2007; Yahia et al. 2017; Novaković et al. 2020), importante para combatir enfermedades producidas por el estrés oxidativo (Valencia-Avilés et al. 2017) como cáncer, enfermedades cardiovasculares y neurodegenerativas, deterioro del sistema inmunológico, enfermedades hepáticas, diabetes mellitus, inflamación, entre otras (Halliwell 1994; Sánchez 2017; Islam et al. 2019).

Debido al gran potencial antioxidante que tienen muchos hongos comestibles silvestres, pueden representar una alternativa natural para prevenir y/o tratar daños causados por el estrés oxidativo, por lo que llevar a cabo investigaciones que ayuden a conocer la concentración y composición de compuestos fenólicos, así como la evaluación de su actividad antioxidante cobra cada día más relevancia. No obstante, a pesar de que México es un país con gran tendencia micófaga, los estudios realizados sobre la composición química y actividad biológica de hongos comestibles silvestres aún son escasos. Al respecto se ha determinado la concentración de compuestos fenólicos totales y se ha evaluado la actividad antioxidante de los hongos comestibles silvestres: Agaricus sp., Boletus sp., Macrolepiota sp. (Alvarez-Parrilla et al. 2007), Amanita caesarea, Cantharellus cibarius, Ramaria sp. (López-Vázquez et al. 2017), Hygrophorus sordidus, Agaricus arvensis, Boletus frostii, Ramaria flava, Lycoperdon perlatum, Boletus luridus, Hypomyces lactiflorum (Yahia et al. 2017), Boletus edulis, Lactarius indigo (López-Vázquez et al. 2017; Yahia et al. 2017) y Morchella vulgaris (Moctezuma-Pérez et al. 2017), lo que significa que de las 450 especies de hongos consumidas solo el $3.5 \%$ han sido estudiadas.

El objetivo de este estudio fue determinar la composición química, cuantificar el contenido de fenoles totales y evaluar la actividad antioxidante de seis especies de hongos comestibles silvestres que crecen en el Área de Protección de Flora y Fauna Nevado de Toluca. Este estudio compara la presencia de compuestos bioactivos y la actividad antioxidante en extractos obtenidos con solventes de diferente polaridad.

\section{MATERIALES Y MÉTODOS}

\section{Material biológico}

Las especies de hongos comestibles silvestres seleccionadas para llevar a cabo este estudio fueron Amanita rubescens, Flammulina mexicana, Floccularia aff. luteovirens, Gyromitra sp., Morchella sp., y Turbinellus floccosus, obtenidos del Área de Protección de Flora y Fauna Nevado de Toluca, localizado en la porción centro sur del Estado de México, dentro de la Faja Vol-

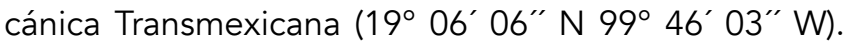
Para esta región se han reportado 77 especies de hongos comestibles silvestres, utilizadas localmente para autoconsumo y venta en mercados regionales (Franco-Maass et al., 2012).

Los esporomas fueron recolectados de junio a septiembre de 2017 y 2018 a partir de un muestreo dirigido con la información obtenida de pobladores locales. Los esporomas recolectados fueron llevados al Laboratorio de Micología del Centro de Investigación 
en Recursos Bióticos de la Universidad Autónoma del Estado de México, donde se realizó la identificación taxonómica de las especies mediante la caracterización macro y micromorfológica con ayuda de las aportaciones de Franco-Maass et al. (2012). Los ejemplares caracterizados fueron depositados en el herbario del mismo laboratorio. Posteriormente se deshidrataron a $65{ }^{\circ} \mathrm{C}$ por $72 \mathrm{~h}$ (Medinilla et al. 2010), y se molieron en un procesador de alimentos Magic Bullet Deluxe ${ }^{\circledR}$ para la obtención de extractos.

\section{Obtención de extractos}

Para la obtención de extractos se utilizaron cuatro solventes de diferente polaridad (agua destilada estéril, metanol, acetato de etilo y hexano), siguiendo el método propuesto por Barros et al. (2007). Se maceraron $10 \mathrm{~g}$ de hongo deshidratado y molido con $100 \mathrm{~mL}$ de cada solvente y se incubaron durante $24 \mathrm{~h}$ con agitación a $25^{\circ} \mathrm{C}$ y $150 \mathrm{rpm}$. Posteriormente, los extractos se filtraron con papel Whatman No. 4 y los residuos se volvieron a extraer dos veces más siguiendo el mismo procedimiento. Finalmente, los extractos de cada especie fúngica se combinaron y se concentraron en rotavapor a $50{ }^{\circ} \mathrm{C}$, a excepción de los extractos acuosos que fueron liofilizados.

A cada uno de los extractos se les practicó un análisis preliminar micoquímico siguiendo el método de Domínguez (1988) para determinar la presencia de flavonoides, saponinas, taninos, quinonas, cumarinas y azucares reductores.

\section{Determinación de compuestos fenólicos totales}

La concentración de compuestos fenólicos totales de los extractos fúngicos se determinó por espectrofotometría, de acuerdo con lo propuesto por Turkoglu et al. (2007), con algunas modificaciones. Se añadió $1 \mathrm{~mL}$ de extracto fúngico $(200 \mu \mathrm{g} / \mathrm{mL})$ a $46 \mathrm{~mL}$ de agua destilada y se le adicionó $1 \mathrm{~mL}$ de reactivo de Folin-Ciocalteu, después de tres minutos de agitación en vortex se adicionaron $3 \mathrm{~mL}$ de $\mathrm{Na}_{2} \mathrm{CO}_{3}$ al 2 \% y se incubó en agitación a temperatura ambiente por 90 min para después medir la absorbancia en un espetrofotómetro a 760 nm (BOECO S-20 Vis, Germany). Todos los ensayos se realizaron por triplicado. La concentración de compuestos fenólicos totales se calculó mediante la ecuación generada por la regresión lineal de la curva de calibración de una solución estándar de ácido gálico $\left(\operatorname{Sigma}^{\circledR}\right.$ ) de $100 \mu \mathrm{g} / \mathrm{mL}$. Los resultados fueron expresados en mg de ácido gálico equivalentes por 100 gramos de hongo seco (mg AGE/100 g hs).

\section{Evaluación de la actividad antioxidante}

La actividad antioxidante se evaluó por el método de captación de la actividad de radicales libres en DPPH (2,2-difenil-1-picrilhidrazil), siguiendo el método de Devi et al. (2008), con algunas modificaciones. Se mezclaron $100 \mu \mathrm{L}$ de cada uno de los extractos fúngicos a diferentes concentraciones $(50,100,150,200$, 250 y $500 \mu \mathrm{g} / \mathrm{mL}$ ) con un $\mathrm{mL}$ de DPPH (Sigma ${ }^{\circledR}$ ) $6 \times 10^{-5}$ $M$ disuelto en metanol, cada reacción se mezcló en vortex y se incubó por 30 min en oscuridad a temperatura ambiente. Posteriormente se midió la absorbancia a $517 \mathrm{~nm}$ en un espectrofotómetro (BOECO S-20 Vis, Germany), utilizando metanol como blanco. Se utilizaron las mismas concentraciones de terbutil hidroquinona (TBHQ) y $\alpha$-tocoferol como controles positivos. La actividad antioxidante se expresó como porcentaje de inhibición lo cual corresponde a la cantidad de radical DPPH neutralizado por el extracto a una determinada concentración, de acuerdo a la ecuación:

$$
\% \text { de inhibición }=\% \mathrm{I}=(\mathrm{A}-\mathrm{A} 1) / \mathrm{A} \times 100
$$

Donde $\mathrm{A}=$ Absorbancia del blanco y $\mathrm{A} 1$ = Absorbancia de la muestra.

Los porcentajes de inhibición obtenidos se graficaron contra la concentración de extracto y se calculó la concentración inhibitoria media (IC50) a partir de la ecuación generada por la regresión logarítmica en el gráfico.

Finalmente, se calculó el coeficiente de correlación ( $r$ ) entre la concentración de compuestos fenólicos totales y la actividad antioxidante.

\section{Análisis estadístico}

Para detectar diferencias entre variables, los datos de concentración de compuestos fenólicos fueron analizados con un ANOVA de una vía y los datos obtenidos de la actividad antioxidante se analizaron con un ANOVA de dos vías, seguido de una prueba a posteriori de comparación de medias de Tukey, utilizando el software Statgraphics ${ }^{\circledR}$ Centurion XVIII (Statpoint Technologies, Inc., 2018). 


\section{RESULTADOS Y DISCUSIÓN}

Análisis preliminar micoquímico

En la Tabla 1 se muestran los resultados del análisis preliminar micoquímico, el cual permitió conocer los metabolitos secundarios presentes en las seis especies de hongos comestibles silvestres investigadas. En extractos acuosos y metanólicos se encontraron la mayoría de los grupos químicos buscados. Al respecto, estudios han reportado que el solvente utilizado para la obtención de extractos tiene un efecto importante en la detección y cuantificación de compuestos bioactivos en hongos (Wang y Xu 2014; Pumtes et al. 2016; Yahia et al. 2017). Se ha encontrado que solventes polares tienen mayor eficiencia de extracción, sugiriendo que la mayoría de los compuestos bioactivos presentes en los hongos tienen polaridad alta (Boonsong et al. 2016).

Como se observa en la Tabla 1, en los diferentes extractos fúngicos existe presencia de saponinas, quinonas y cumarinas. En extractos de F. mexicana y Gyromitra sp., se encontró además la presencia de flavonoides, taninos y azúcares reductores. En algunas especies como A. rubescens, F. aff. luteovirens y Morchella sp. no se detectaron taninos y en las últimas dos especies mencionadas tampoco se encontraron flavonoides. Lo encontrado en este trabajo es el primer reporte de la composición química de las especies de hongos comestibles estudiadas.

\section{Compuestos fenólicos totales}

Los compuestos fenólicos son considerados los principales compuestos antioxidantes en hongos comestibles silvestres (Choi y Sapers 1994; Athanasakis et al. 2013; Wang y Xu 2014), razón por la cual se ha estu- diado su presencia y concentración en diversos hongos, con la finalidad de encontrar fuentes naturales de antioxidantes o compuestos que puedan ser utilizados como blancos farmacéuticos (Elmastas et al. 2007; Soares et al. 2009; Kozarski et al. 2015; Yahia et al. 2017; Islam et al. 2019).

Para todos los hongos comestibles silvestres estudiados, los extractos obtenidos con agua y metanol exhiben concentraciones de fenoles totales significativamente más altas que los extractos obtenidos con acetato de etilo y hexano. Los resultados presentados en la Tabla 2 muestran que la mayor concentración de compuestos fenólicos se encuentra en extractos acuosos de Morchella sp. (19.86 mg AGE/100g hs), Gyromitra sp. (18.65 mg AGE/100g hs) y F. aff. luteovirens (17.84 mg AGE/100g hs), con diferencias estadísticas significativas $(p<0.05)$ con el resto de los extractos, mientras que la menor concentración se encuentra en extractos acetílicos de $T$. floccosus (3.20 mg AGE $1100 \mathrm{~g}$ hs) $\left(\mathrm{F}_{27,83}=220.69, \mathrm{p} \leq 0.0000\right)$. Al respecto, Wang y Xu (2014), encontraron que extractos acuosos de 20 hongos comestibles de China tienen valores significativamente más altos de fenoles totales que otros solventes como acetona y etanol.

En este trabajo se presenta por primera vez la cuantificación de fenoles totales para las seis especies de hongos comestibles de estudio en el Estado de México. Al respecto, Kouassi et al. (2016), estudiaron el contenido de compuestos fenólicos de $A$. rubescens en Costa de Marfil, encontrando una concentración de fenoles

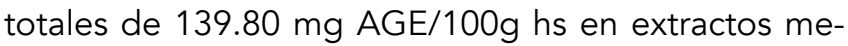
tanólicos, mientras que en este estudio en la misma especie se encontraron $10.54 \mathrm{mg} \mathrm{AGE/100g} \mathrm{hs} \mathrm{en}$ extractos obtenidos con el mismo solvente. Estudios sugieren que la biogénesis de metabolitos secundarios

Tabla 1. Análisis preliminar micoquímico de extractos de seis especies de hongos comestibles silvestres

\begin{tabular}{|c|c|c|c|c|c|c|}
\hline \multirow[b]{2}{*}{ Especie } & \multicolumn{6}{|c|}{ Grupo químico ${ }^{1}$} \\
\hline & Flavonoides & Saponinas & Taninos & Quinonas & Cumarinas & $\begin{array}{l}\text { Azúcares } \\
\text { reductores }\end{array}$ \\
\hline Amanita rubescens & Ac & $\mathrm{Ac} / \mathrm{M} / \mathrm{AE} / \mathrm{H}$ & nd & $\mathrm{Ac} / \mathrm{M} / \mathrm{H}$ & $\mathrm{Ac} / \mathrm{M} / \mathrm{AE} / \mathrm{H}$ & $\mathrm{Ac} / \mathrm{M} / \mathrm{H}$ \\
\hline Flammulina mexicana & M & $\mathrm{M} / \mathrm{AE} / \mathrm{H}$ & Ac & $\mathrm{Ac} / \mathrm{M} / \mathrm{H}$ & $\mathrm{M} / \mathrm{AE}$ & $\mathrm{Ac} / \mathrm{M} / \mathrm{H}$ \\
\hline Floccularia aff. luteovirens & nd & $\mathrm{AE}$ & nd & $\mathrm{Ac} / \mathrm{M} / \mathrm{H}$ & $A E$ & $\mathrm{Ac} / \mathrm{M}$ \\
\hline Gyromitra sp. & Ac & $\mathrm{Ac} / \mathrm{M} / \mathrm{AE} / \mathrm{H}$ & $\mathrm{Ac} / \mathrm{M} / \mathrm{AE}$ & $\mathrm{H}$ & $\mathrm{Ac} / \mathrm{M} / \mathrm{AE} / \mathrm{H}$ & $\mathrm{Ac} / \mathrm{M} / \mathrm{H}$ \\
\hline Morchella sp. & nd & $\mathrm{AE}$ & nd & $\mathrm{Ac} / \mathrm{H}$ & $\mathrm{Ac} / \mathrm{AE}$ & nd \\
\hline Turbinellus floccosus & $\mathrm{Ac} / \mathrm{M}$ & $\mathrm{Ac} / \mathrm{M} / \mathrm{AE} / \mathrm{H}$ & $\mathrm{Ac} / \mathrm{M} / \mathrm{AE}$ & $\mathrm{Ac} / \mathrm{M} / \mathrm{H}$ & $\mathrm{Ac} / \mathrm{M} / \mathrm{AE} / \mathrm{H}$ & nd \\
\hline
\end{tabular}

${ }_{1}$ Grupo químico detectado ( $\mathrm{Ac}=$ =agua, $\mathrm{M}=$ metanol, $\mathrm{AE}=$ =acetato de etilo, $\mathrm{H}=$ hexano) o no detectado (nd). 
Tabla 2. Concentración de compuestos fenólicos totales de seis especies de hongos comestibles silvestres

\begin{tabular}{|c|c|c|c|c|}
\hline \multirow{2}{*}{ Especie } & \multicolumn{4}{|c|}{ Compuestos fenólicos totales (mg AGE/100g hs) ${ }^{1}$} \\
\hline & Agua & Metanol & Acetato de etilo & Hexano \\
\hline Amanita rubescens & $11.82 \pm 1.00 \mathrm{~A}, \mathrm{~d}$ & $10.54 \pm 0.77 \mathrm{~A}$, def & $4.32 \pm 0.55 \mathrm{~B}, \mathrm{bc}$ & $4.36 \pm 0.55 \mathrm{~B}, \mathrm{~d}$ \\
\hline Flammulina mexicana & $16.64 \pm 0.45 \mathrm{~A}, \mathrm{bc}$ & $12.78 \pm 0.51 \mathrm{~B}, \mathrm{bc}$ & $4.73 \pm 0.35 \mathrm{D}, \mathrm{bc}$ & $8.13 \pm 0.41 \mathrm{C}, \mathrm{b}$ \\
\hline Floccularia aff. luteovirens & $17.84 \pm 0.40 \mathrm{~A}, \mathrm{~b}$ & $14.87 \pm 0.30 \mathrm{~B}, \mathrm{a}$ & $3.6 \pm 0.68 \mathrm{D}, \mathrm{C}$ & $7.97 \pm 0.20 \mathrm{C}, \mathrm{b}$ \\
\hline Gyromitra sp. & $18.65 \pm 0.45 \mathrm{~A}, \mathrm{ab}$ & $11.98 \pm 0.36 \mathrm{~B}, \mathrm{~cd}$ & $5.77 \pm 0.56 \mathrm{C}, \mathrm{ab}$ & $6.61 \pm 0.61 \mathrm{c}, \mathrm{bc}$ \\
\hline Morchella sp. & $19.86 \pm 1.00 \mathrm{~A}, \mathrm{a}$ & $13.59 \pm 0.75 \mathrm{~B}, \mathrm{ab}$ & $6.89 \pm 1.17 \mathrm{D}, \mathrm{a}$ & $8.85 \pm 1.04 \mathrm{C}, \mathrm{a}$ \\
\hline Turbinellus floccosus & $9.25 \pm 0.55 \mathrm{~B}, \mathrm{e}$ & $11.34 \pm 0.35 \mathrm{~A}, \mathrm{cde}$ & $3.20 \pm 0.20 \mathrm{D}, \mathrm{C}$ & $5.96 \pm 0.35 \mathrm{C}, \mathrm{cd}$ \\
\hline
\end{tabular}

Datos expresados como medias \pm SD de tres repeticiones. Medias con diferente letra mayúscula (A-D) en la misma fila son significativamente diferentes $(p<0.05$, ANOVA, Tukey-HSD). Medias con diferente letra minúscula (a-f) en la misma columna son significativamente diferentes ( $p<0.05$, ANOVA, Tukey-HSD).

en hongos está condicionada principalmente por el tipo de nutrientes que conforman el sustrato y las condiciones climáticas que los rodean, por lo que la región y los diferentes sustratos donde crecen y se desarrollan los hongos pueden alterar y cambiar su metabolismo, generando diferencias en la presencia de compuestos bioactivos y su concentración (Jong y Birmingham 1992; Sánchez 2017)

A pesar de que en el análisis micoquímico de Morchella sp. sólo se detectaron tres grupos químicos, en el ensayo de Folin-Ciocalteu este hongo tiene las concentraciones más altas de fenoles totales en tres de los cuatro solventes utilizados con 19.86, 6.89 y 8.85 mg AGE/100g hs en extractos en agua, acetato de etilo y hexano respectivamente. Esto es importante si se considera que el género Morchella es uno de los más apreciados por su alta demanda en el mercado internacional tanto por su sabor, como por sus propiedades nutricionales y funcionales (Moctezuma-Pérez et al. 2017), lo cual resalta con los resultados obtenidos en este estudio. Gursoy et al. (2009), estudiaron el contenido de fenoles totales de siete especies del género Morchella en Turquía, encontrando la mayor concentración en extractos metanólicos de $M$. conica (25.38 $\mu \mathrm{g}$ AGE/mg extracto) y la menor concentración en $M$. deliciosa (12.36 $\mu \mathrm{g}$ AGE/mg extracto). Para el resto de las especies no se reportan valores, por lo que el presente estudio es el primer reporte sobre concentración de compuestos fenólicos totales.

\section{Actividad antioxidante}

Los antioxidantes son compuestos capaces de inhibir la iniciación y/o propagación de las reacciones en ca- dena de los radicales libres, causantes de daño oxidativo que juegan un papel importante en diversas enfermedades como el cáncer, además de estar asociados al proceso de envejecimiento (Valko et al. 2007; Athanasakis et al. 2013; Islam et al. 2019). Los antioxidantes presentes en la dieta tienen gran relevancia al considerarse elementos benéficos e indispensables para la salud. Es así como los hongos cobran importancia y han sido objeto de numerosas investigaciones que correlacionan la concentración de compuestos fenólicos con la actividad antioxidante in vitro (Cheung et al. 2003; Athanasakis et al. 2013; Sánchez y Liedo 2017). De la diversidad de métodos para evaluar la actividad antioxidante, el más empleado en hongos ha sido el de captación de radicales libres DPPH, que con diversas modificaciones ha demostrado que los hongos son excelentes antioxidantes (Sánchez y Liedo 2017).

En este trabajo se midió la actividad antioxidante de los extractos fúngicos y dos controles (TBHO y $\alpha$-tocoferol) a seis diferentes concentraciones de 50 a $500 \mu \mathrm{g} /$ $\mathrm{mL}$. En la Figura 1 se observa que los porcentajes de inhibición aumentan a medida que aumenta la concentración de extracto. La mayor actividad antioxidante se encontró en extractos acuosos y metanólicos con porcentajes de inhibición mayores al control TBHO pero menores al control $\alpha$-tocoferol. Extractos hexánicos y en acetato de etilo mostraron la menor actividad antioxidante con porcentajes de inhibición menores que ambos controles.

En la Tabla 3 se muestran los resultados expresados como porcentaje de inhibición en la concentración más alta de extracto $(500 \mu \mathrm{g} / \mathrm{mL})$. En extractos acuosos, Morchella sp. (81.3 \%), Gyromitra sp. (78.2 \%) y 


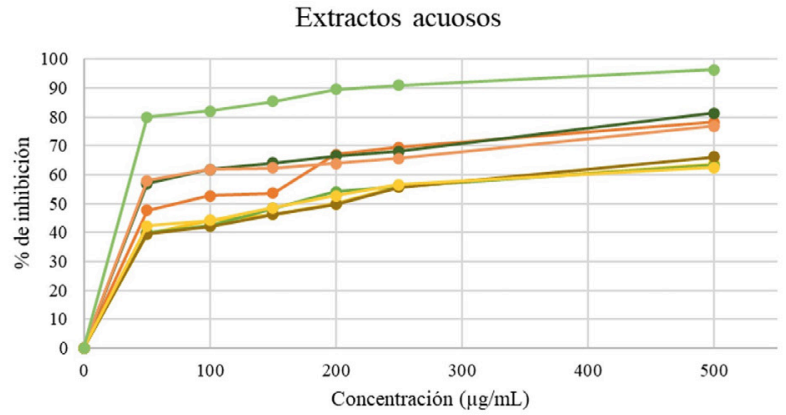

Extractos en acetato de etilo
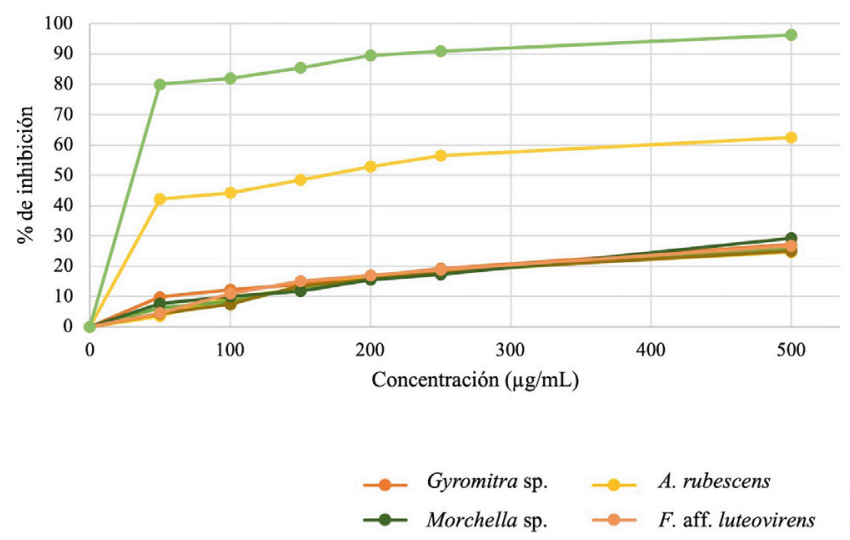

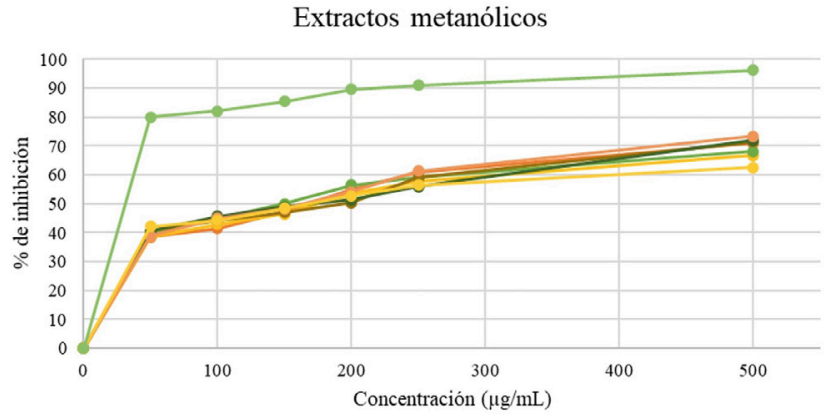

Extractos hexánicos
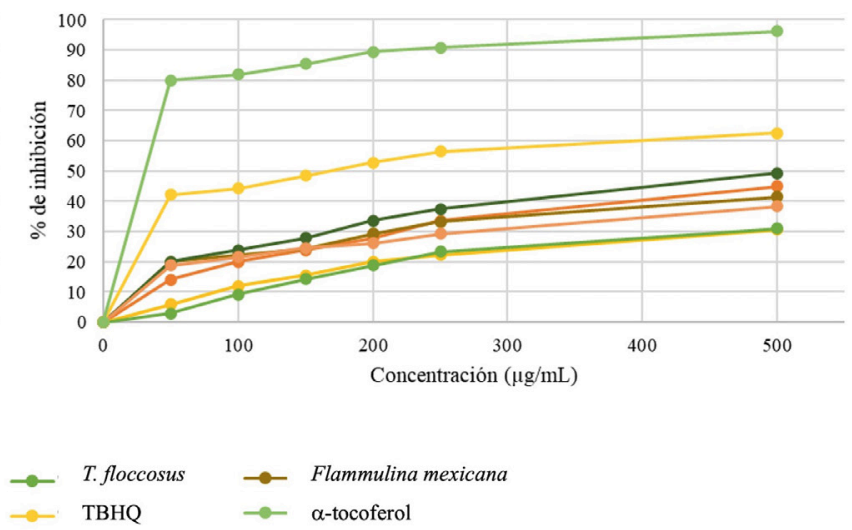

Figura 1. Evaluación de la actividad antioxidante por el método DPPH. Porcentaje de inhibición de seis concentraciones $(50-500 \mu \mathrm{g} / \mathrm{mL})$ de extracto fúngico, terbutil hidroquinona (TBHQ) y $\alpha$-tocoferol.

Tabla 3. Actividad antioxidante de seis especies de hongos comestibles silvestres

\begin{tabular}{|c|c|c|c|c|}
\hline \multirow{2}{*}{ Especie } & \multicolumn{4}{|c|}{ Actividad antioxidante (\% de inhibición) ${ }^{1}$} \\
\hline & Agua & Metanol & Acetato de etilo & Hexano \\
\hline Amanita rubescens & $63.7 \pm 0.00 \mathrm{~B}, \mathrm{de}$ & $66.8 \pm 0.00 \mathrm{~A}, \mathrm{bc}$ & $24.7 \pm 0.00 \mathrm{D}, \mathrm{de}$ & $30.7 \pm 0.00 \mathrm{C}, \mathrm{g}$ \\
\hline Flammulina mexicana & $66.1 \pm 0.00 \mathrm{~B}, \mathrm{~d}$ & $71.5 \pm 0.00 \mathrm{~A}, \mathrm{~b}$ & $25.0 \pm 0.00 \mathrm{D}, \mathrm{de}$ & $41.5 \pm 0.00 \mathrm{C}, \mathrm{de}$ \\
\hline Floccularia aff. luteovirens & $76.8 \pm 0.00 \mathrm{~A}, \mathrm{bc}$ & $73.3 \pm 0.00 \mathrm{~B}, \mathrm{~b}$ & $26.8 \pm 0.00 \mathrm{D}, \mathrm{cd}$ & $38.3 \pm 0.00 \mathrm{C}, \mathrm{ef}$ \\
\hline Gyromitra sp. & $78.2 \pm 0.00 \mathrm{~A}, \mathrm{bc}$ & $71.0 \pm 0.00 \mathrm{~B}, \mathrm{~b}$ & $27.2 \pm 0.00 \mathrm{D}, \mathrm{cd}$ & $45.0 \pm 0.00 \mathrm{C}, \mathrm{d}$ \\
\hline Morchella sp. & $81.3 \pm 0.00 \mathrm{~A}, \mathrm{~b}$ & $72.0 \pm 0.00 \mathrm{~B}, \mathrm{~b}$ & $29.2 \pm 0.00 \mathrm{D}, \mathrm{C}$ & $49.3 \pm 0.00 \mathrm{C}, \mathrm{c}$ \\
\hline Turbinellus floccosus & $63.1 \pm 0.00 \mathrm{~B}, \mathrm{de}$ & $68.2 \pm 0.00 \mathrm{~B}, \mathrm{bc}$ & $25.8 \pm 0.00 \mathrm{D}, \mathrm{de}$ & $31.0 \pm 0.00 \mathrm{C}, \mathrm{g}$ \\
\hline TBHO & $62.5 \pm 0.00 \mathrm{de}$ & $62.5 \pm 0.00 \mathrm{de}$ & $62.5 \pm 0.00 \mathrm{~b}$ & $62.5 \pm 0.00 \mathrm{~b}$ \\
\hline$\alpha$-tocoferol & $96.3 \pm 0.00 \mathrm{a}$ & $96.3 \pm 0.00 \mathrm{a}$ & $96.3 \pm 0.00 \mathrm{a}$ & $96.3 \pm 0.00 \mathrm{a}$ \\
\hline
\end{tabular}

${ }^{1}$ Datos expresados como medias \pm SD de tres repeticiones a una concentración de $500 \mu \mathrm{g} / \mathrm{mL}$. Medias con diferente letra mayúscula (A-D) en la misma fila son significativamente diferentes ( $p<0.05$, ANOVA, Tukey-HSD). Medias con diferente letra minúscula (a-g) en la misma columna son significativamente diferentes $(p<0.05$, ANOVA, Tukey-HSD). Terbutil hidroquinona (TBHQ) y $\alpha$-tocoferol como controles. 
F. aff. luteovirens $(76.8 \%)$ tienen la mayor actividad antioxidante. En extractos metanólicos, F. aff. luteovirens $(73.3 \%)$ tuvo el mayor porcentaje de inhibición, seguido de Morchella sp. (72 \%) y F. mexicana (71.5 \%). El porcentaje de inhibición del resto de las especies es comparable estadísticamente con la del control TBHQ (62.5\%).

Extractos en acetato de etilo y hexano presentaron porcentajes de inhibición menores a ambos controles. Sin embargo, los extractos hexánicos tuvieron mayor actividad antioxidante que los acetílicos con diferencias estadísticamente significativas $(p<0.05)$. Morchella sp. tuvo la mayor actividad antioxidante con porcentajes de inhibición de $49.3 \%$ en hexano y $29.2 \%$ en acetato de etilo, mientras que $A$. rubescens tuvo los porcentajes de inhibición más bajos para extractos hexánicos (30.7 \%) y acetílicos (24.7 \%) $\left(F_{167,503}=315.98\right.$, $\mathrm{p} \leq 0.0000)$.

Existe una correlación positiva $(r=0.925)$ entre la concentración de fenoles totales y la actividad antioxidante, al ser los extractos acuosos de Morchella sp., Gyromitra sp. y F. aff. luteovirens los que presentaron la mayor concentración de compuestos fenólicos totales y los mayores porcentajes de inhibición en el ensayo de actividad antioxidante in vitro DPPH, mientras que los extractos de hexano y acetato de etilo de $A$. rubescens presentan las concentraciones más bajas de fenoles totales y actividad antioxidante.

En la Tabla 4 se compara la concentración inhibitoria media (IC50), que corresponde a la concentración de extracto necesaria para inhibir el $50 \%$ del radical DPPH. Los extractos acuosos y metanólicos presentan valores de IC50 menores o iguales a $200 \mu \mathrm{g} / \mathrm{mL}$, por el contrario, los valores de IC50 de extractos en acetato de etilo y hexano exceden los $500 \mu \mathrm{g} / \mathrm{mL}$. Extractos acuosos de $T$. floccosus $(160 \mu \mathrm{g} / \mathrm{mL})$ y extractos metanólicos de T. floccosus $(150 \mu \mathrm{g} / \mathrm{mL})$, Morchella sp. (160 $\mu \mathrm{g} / \mathrm{mL}$ ) y $F$. aff. luteovirens $(162 \mu \mathrm{g} / \mathrm{mL})$, presentan valores de IC50 iguales o cercanos a los del control TBHO $(160 \mu \mathrm{g} / \mathrm{mL})$. Los extractos acuosos de Morchella sp., F. aff. luteovirens y Gyromitra sp., con IC50 de $41 \mu \mathrm{g} / \mathrm{mL}$, $42 \mu \mathrm{g} / \mathrm{mL}$ y $78 \mu \mathrm{g} / \mathrm{mL}$ respectivamente resultan ser los más promisorios desde el punto de vista de su actividad antioxidante al tener los IC50 más bajos.

Sobresale el potencial antioxidante de Morchella sp., que tuvo la mayor concentración de fenoles totales, el porcentaje de inhibición más alto por medio de la reacción de DPPH y el menor IC50 en tres de los cuatro solventes utilizados, siendo el agua, el solvente de extracción más eficiente para este ensayo. Al

Tabla 4. Concentración Inhibitoria media (IC50) obtenida para cada extracto evaluado por el método de captación de radicales libres DPPH

\begin{tabular}{|c|c|c|c|c|}
\hline \multirow{2}{*}{ Especie } & \multicolumn{4}{|c|}{ IC50 $(\mu \mathrm{g} / \mathrm{mL})^{1}$} \\
\hline & Agua & Metanol & Acetato de etilo & Hexano \\
\hline Amanita rubescens & 200 & 180 & 7332 & 3181 \\
\hline Flammulina mexicana & 200 & 200 & 7332 & 1517 \\
\hline Floccularia aff. luteovirens & 42 & 162 & 6003 & 2728 \\
\hline Gyromitra sp. & 78 & 175 & 14765 & 871 \\
\hline Morchella sp. & 41 & 160 & 7332 & 650 \\
\hline Turbinellus floccosus & 160 & 150 & 9897 & 2301 \\
\hline TBHO & 160 & 160 & 160 & 160 \\
\hline$\alpha$-tocoferol & 25 & 25 & 25 & 25 \\
\hline
\end{tabular}

${ }^{1}$ Valores obtenidos a partir de la ecuación generada por la regresión logarítmica en el gráfico de porcentaje de inhibición contra concentración de extracto del ensayo DPPH ( $\left.\mathrm{R}^{2}=0.98\right)$. Terbutil hidroquinona (TBHQ) y $\alpha$-tocoferol como controles. 
respecto, Moctezuma-Pérez et al. (2017) evaluaron las propiedades nutracéuticas incluyendo la actividad antioxidante de extractos acuosos y metanólicos de la especie mexicana Morchella vulgaris encontrando mayor actividad antioxidante en extractos acuosos con un porcentaje de $43.23 \%$, por lo que el porcentaje de $81.3 \%$ encontrado en este trabajo es significativamente mayor.

Para A. rubescens se han encontrado porcentajes de inhibición de 67.17 \% (Kouassi et al. 2016) y de 91.31 \% (Keles et al. 2011) en extractos metanólicos, mientras que en el presente, el porcentaje para la misma especie en extractos metanólicos es de $66.8 \%$. Para el resto de las especies, no se reportan estudios previos, ya que la mayoría de los trabajos están enfocados en conocer y evaluar la actividad antioxidante y otras propiedades medicinales y nutricionales de los principales hongos comestibles y medicinales cultivados, dejando de lado el estudio de las especies silvestres a pesar de que en el centro de México son utilizadas tradicionalmente como alimento y medicina, o bien, como una fuente de ingresos en la temporada de lluvias.

Especies como A. rubescens, T. floccosus, Gyromitra sp., y Morchella sp. forman parte de la dieta y del conocimiento micológico tradicional de los habitantes de diferentes municipios de los Estados de México (Mariaca et al. 2001; Estrada-Martínez et al. 2009; Aguilar-Cruz y Villegas 2010; Burrola-Aguilar et al. 2012), Oaxaca (Garibay-Orijel et al. 2009; Zamora-Martínez et al. 2014) y Tlaxcala (Montoya et al. 2019) por mencionar algunos, lo que evidencia la necesidad de continuar con estudios más profundos de la composición química de estas especies, que permitan encontrar moléculas con posibles propiedades medicinales y funcionales.

Es de interés mencionar al género Gyromitra, cuya comestibilidad ha causado controversia. Algunas especies son consideradas tóxicas, por la producción del compuesto giromitrina y debido a su difícil identificación, muchas veces son mezcladas las especies tóxicas y no tóxicas (Lima et al. 2012). No obstante, evidencia reciente cataloga a las especies de este género como comestibles bajo ciertas condiciones (Li et al. 2021), ya que la giromitrina es una molécula soluble en agua y volátil, los hongos pueden ser consumidos sin riesgo de intoxicación al ser hervidos en agua durante mucho tiempo y permitiendo su secado (Lima et al. 2012), técnica que forma parte del conocimiento tradicional de las personas que consumen este hongo. Por lo que la alta actividad antioxidante que presentó esta especie puede ser aprovechada.

Adicionalmente, las especies de hongos comestibles silvestres Flammulina mexicana y Floccularia aff. Iuteovirens del Área de Protección de Flora y Fauna Nevado de Toluca, han sido estudiadas con fines de cultivo experimental (Arana-Gabriel et al. 2014, 2019, 2020), con la obtención de fructificaciones de F. mexicana (Arana-Gabriel et al. 2020), por lo que el conocimiento de sus propiedades antioxidantes da un valor agregado al consumo de estas especies y permite ampliar el número de hongos comestibles cultivados para una futura comercialización y contar con nuevas alternativas que permitan promover el consumo de hongos.

\section{CONCLUSIÓN}

Químicamente, los hongos comestibles silvestres estudiados: Amanita rubescens, Flammulina mexicana, Floccularia aff. luteovirens, Gyromitra sp., Morchella sp., y Turbinellus floccosus presentan flavonoides, saponinas, quinonas, cumarinas y azucares reductores. Solventes de elevada polaridad como el agua y el metanol resultan ser más eficientes que el hexano y el acetato de etilo para la extracción de compuestos bioactivos polares en hongos. Ya que es en este tipo de solventes donde se encuentran las concentraciones más altas de compuestos fenólicos totales y la mayor actividad antioxidante.

Los extractos acuosos de Morchella sp., Gyromitra sp. y Floccularia aff. luteovirens resultaron ser los más promisorios al presentar las mayores concentraciones de compuestos fenólicos totales, la mayor actividad antioxidante in vitro y los valores más bajos de IC50 por lo que pueden ser considerados como una potencial fuente de antioxidantes naturales.

En México, los estudios sobre la composición química y las propiedades antioxidantes de hongos comestibles silvestres aún son muy escasos y los resultados de este trabajo contribuyen al conocimiento de la actividad biológica de los compuestos bioactivos presentes en hongos silvestres. Tomando en cuenta los resultados obtenidos, estos hongos pueden ser considerados como complemento de la dieta, ya que al tener importantes propiedades antioxidantes pueden presentar beneficios en la salud de quien los consume. 


\section{AGRADECIMIENTOS}

Los autores agradecen a la Secretaría de Educación Pública y a la Universidad Autónoma del Estado de México por el financiamiento de este proyecto, a través del Apoyo para Fortalecimiento de Cuerpos Académicos en Formación del Programa para el Desarrollo Profesional Docente de la Secretaría de Educación Pública (PRODEP-SEP) 2018 (Proyecto No. 4615/2018 CA: "Avances etnofarmacológicos, nutricionales y nutricéuticos de los recursos vegetales y fúngicos prioritarios para las comunidades del Santuario de Agua Presa Corral de Piedra) y a la Secretaría de Investigación y Posgrado del Instituto Politécnico Nacional por parte del financiamiento de este trabajo a través del Proyecto SIP-IPN 2021163, así como al Consejo Nacional de Ciencia y Tecnología (CONACyT) por la beca otorgada a la primera autora para sus estudios en el Posgrado en Ciencias en Biomedicina y Biotecnología Molecular de la Escuela Nacional de Ciencias Biológicas de Instituto Politécnico Nacional.

\section{LITERATURA CITADA}

Aguilar-Cruz Y, Villegas M. 2010. Gomphales comestibles en el municipio de Villa del Carbón Estado de México. Revista Mexicana de Micología 31, 1-8.

Alvarez-Parrilla E, De la Rosa LA, Martínez NR, González-Aguilar GA. 2007. Total phenols and antioxidant activity of commercial and wild mushrooms from Chihuahua, Mexico. Ciencia y Tecnología Alimentaria 5, 329-334. https://doi.org/10.1080/11358120709487708

Arana-Gabriel Y, Burrola-Aguilar C, Garibay-Orijel R. Franco-Maass S. 2014. Obtención de cepas y producción de inóculo de cinco especies de hongos silvestres comestibles de alta montaña en el centro de México. Revista Chapingo Serie Ciencias Forestales y del Ambiente 20, 213-226. https://doi.org/10.5154/r.rchscfa.2014.04.017

Arana-Gabriel Y, Burrola-Aguilar C, Franco-Maass S, Mata G, Zepeda-Gómez C. 2019. Colonización miceliar de Flammulina mexicana a partir de inóculo sólido y líquido en residuos agroforestales. Revista Mexicana de Ciencias Agricolas 10, 11-22. https://doi. org/10.29312/remexca.v10i1.376

Arana-Gabriel Y, Burrola-Aguilar C, Franco-Maass S, Mata G, Zepeda-Gómez C. 2020. Characterization and evaluation of Flammulina mexicana growth in lignocellulosic residues. Bosque 41, 173182. http://dx.doi.org/10.4067/S0717-92002020000200173

Athanasakis G, Aligiannis N, Gonou-Zagou Z, Skaltsounis AL, Fokialakis N. 2013. Antioxidant properties of the wild edible mushroom Lactarius salmonicolor. Journal of Medicinal Food 16, 760-764. https://doi.org/10.1089/jmf.2012.0297

Barros L, Calhelha RC, Vaz JA, Ferreira ICFR, Baptista P, Estevinho LM. 2007. Antimicrobial activity and bioactive compounds of Portuguese wild edible mushrooms methanolic extracts. European Food Research Technology 225, 151-156. https://doi. org/10.1007/s00217-006-0394-x

Bautista-González J.A, Herrera-Campos MA. 2019. Hongos y líquenes en la medicina tradicional mexicana. Arqueología Mexicana 87, 62-65.

Boonsong S, Klaypradit W, Wilaipuna P. 2016. Antioxidant activities of extracts from five edible mushrooms using different extractants. Agriculture and Natural Resources 50, 89-97. https://doi. org/10.1016/j.anres.2015.07.002
Burrola-Aguilar C, Montiel O, Garibay-Orijel R, Zizumbo-Villarreal L. 2012. Conocimiento tradicional y aprovechamiento de los hongos comestibles silvestres en la región de Amanalco, Estado de México. Revista Mexicana de Micología 35, 1-16.

Chaturvedi VK, Agarwal S, Gupta KK, Ramteke PW, Singh MP. 2018 Medicinal mushroom: boon for therapeutic applications. Biotech 8, 1-20. https://doi.org/10.1007/s13205-018-1358-0

Cheung LM, Cheung PCK, Ooi VEC. 2003. Antioxidant activity and total phenolics of edible mushroom extracts. Food Chemistry 81, 249-255. https://doi.org/10.1016/S0308-8146(02)00419-3

Choi SW, Sapers GM. 1994. Effects of washing on polyphenols and polyphenol oxidase in commercial mushrooms (Agaricus bisporus). Journal of Agricultural and Food Chemistry 42, 2286-2290. https://doi.org/10.1021/jf00046a038

Devi KP, Suganthy N, Kesika P, Pandian SK. 2008. Bioprotective properties of seaweeds: in vitro evaluation of antioxidant activity and antimicrobial activity against food borne bacteria. BMC Complementary and Alternative Medicine 8, 1-11. https://doi. org/10.1186/1472-6882-8-38

Domínguez AX. 1988. Métodos de investigación fitoquímica. Limusa. México, DF.

Elmastas M, Isildak O, Turkekul I, Temur N. 2007. Determination of antioxidant activity and antioxidant compounds in wild edible mushrooms. Journal of Food Composition and Analysis 20, 337-345. https://doi.org/10.1016/j.jfca.2006.07.003

Estrada-Martínez E, Guzmán G, Cibrián-Tovar D, Ortega-Paczka R. 2009. Contribución al conocimiento etnomicológico de los hongos comestibles silvestres de mercados regionales y comunidades de la Sierra Nevada (México). Interciencia 34, 25-33.

Franco-Maass S, Burrola-Aguilar C, Arana-Gabriel Y. 2012. Hongos comestibles silvestres: Un recurso forestal no maderable del Nevado de Toluca. Ediciones EON, México, D.F.

Garibay-Orijel R, Martínez-Ramos M, Cifuentes J. 2009. Disponibilidad de esporomas de hongos comestibles en los bosques de pino-encino de Ixtlán de Juárez, Oaxaca. Revista Mexicana de Biodiversidad 80, 521-534.

Gebreyohannes G, Nyerere A, Bii C, Sbhatu DB. 2019. Determination of antimicrobial activity of extracts of indigenous wild mushrooms against pathogenic organisms. Evidence-Based Complementary and Alternative Medicine Volume 2019, Article ID 6212673. https://doi.org/10.1155/2019/6212673

Golak-Siwulska I, Kałużewicz A, Spiżewski T, Siwulski M, Sobieralski K. 2018. Bioactive compounds and medicinal properties of Oyster mushrooms (Pleurotus sp.). Folia Horticulturae 30, 191-201. https://doi.org/10.2478/fhort-2018-0012

Guillamón S, García-Lafuente A, Lozano M, D’Arrigo M, Rostagno MA, Villares A, Martínez JA. 2010. Edible mushrooms: role in the prevention of cardiovascular diseases. Fitoterapia 81, 715-723. https://doi.org/10.1016/j.fitote.2010.06.005

Gursoy N, Sarikurkcu C, Cengiz M, Solak MH. 2009. Antioxidant activities, metal contents, total phenolics and flavonoids of seven Morchella species. Food and Chemical Toxicology 47, 2381-2388. https://doi.org/10.1016/j.fct.2009.06.032

Halliwell B. 1994. Free radicals, antioxidants, and human disease: curiosity, cause, or consequence? The Lancet 344 (8924), 721-724. https://doi.org/10.1016/s0140-6736(94)92211-x 
Islam T, Ganesan K, Xu B. 2019. New insight into mycochemical profiles and antioxidant potential of edible and medicinal mushrooms: A Review. International Journal of Medicinal Mushrooms 21, 237-251. https://doi.org/10.1615/IntJMedMushrooms. 2019030079

Jong SC, Birmingham JM. 1992. Medicinal benefits of the mushrooms Ganoderma. Advances in Applied Microbiology 37, 101-134. https://doi.org/10.1016/S0065-2164(08)70253-3

Keleș A, Koca I, Gençcelep H. 2011. Antioxidant properties of wild edible mushrooms. Journal Food Processing and Technology 2, 1-6. https://doi.org/10.4172/2157-7110.1000130

Korzaski M, Klaus A, Vunduk J, Zizak Z, Niksic M, Jakovljevic D, Miroslav MV, Leo JLDVG. 2015. Nutraceutical properties of the methanolic extract of edible mushroom Cantharellus cibarius (Fries): primary mechanisms. Food and Function 6, 1875-1886. https:// doi.org/10.1039/c5fo00312a

Kouassi KA, Kouadio EJP, Konan KH, Dué AE, Kouamé LP. 2016. Phenolic compounds, organic acid and antioxidant activity of Lactarius subsericatus, Cantharellus platyphyllus and Amanita rubescens, three edible ectomycorrhizal mushrooms from center of Côte D'ivoire. Eurasian Journal of Analytical Chemistry 11, 127-139.

Li H, Tian Y, Menolli N Jr, Ye L, Karunarathna SC, Perez-Moreno J, Rahman MM, Rashid MH, Phengsintham P, Rizal L, Kasuya T, Lim YW, Dutta AK, Khalid AN, Huyen LT, Balolong MP, Baruah G, Madawala S, Thongklang N, Hyde KD, Kirk PM, Xu J, Sheng J, Boa E, Mortimer PE. 2021. Reviewing the world's edible mushroom species: A new evidence-based classification system. Comprehensive Reviews in Food Science and Food Safety 20, 1982-2014. https:// doi.org/10.1111/1541-4337.12708

Lima AD, Costa Fortes R, Carvalho Garbi Novaes MR, Percário S. 2012. Poisonous mushrooms: a review of the most common intoxications. Nutrición Hospitalaria 27, 402-408. https://doi. org/10.1590/S0212-16112012000200009

López-Vázquez E, Prieto-García F, Gayosso-Canales M, Otazo-Sánchez EM Villagómez-lbarra JR. 2017. Phenolic acids, flavonoids, ascorbic acid, $\beta$-glucans and antioxidant activity in Mexican wild edible mushrooms. Italian Journal of Food Science 29, 766-774. https://doi.org/10.14674/IJFS-838

Mariaca R, Silva-Pérez LC, Castaños-Montes CA. 2001. Proceso de recolección y comercialización de hongos comestibles silvestres en el Valle de Toluca, México. Ciencia Ergo Sum 8, 30-40.

Martínez-Medina GA, Chávez-González ML, Verma DK, Prado-Barragán LA, Martínez-Hernández JL, Flores-Gallegos AC, Thakur M, SrivastavPP, Aguilar CN. 2021. Bio-funcional components in mushrooms, a health opportunity: Ergothionine and huitlacoche as recent trends. Journal of Functional Foods 77, 1-17. https://doi. org/10.1016/j.jff.2020.104326

Medinilla B, Cruz S, Navas G, Arriaga L, Morales O, Cáceres R. 2010. Evaluación biológica y fitoquímica de cinco hongos nativos de Guatemala. Guatefarma.

Moctezuma-Pérez I, Martínez-García M, Campos-Contreras JE, Rivera-Yáñez $N$, Juárez-Contreras $A$, Hernández-Portilla LB, Flores-Ortiz $C M$, Hernández-Hernández $A B$, Martínez-Elizalde KS, Rodríguez MG, Rivera-Yáñez CR, Rodríguez-Monroy MA Canales-Martínez MM. 2017. Propiedades nutracéuticas de Morchella vulgaris (Pers.) Boud. Interciencia 42, 423-429.
Montoya A, Briones-Dumas E, Núñez-López RA, Kong A, Ortíz-Hernández V, Moreno-Fuentes A. 2019. Los hongos conocidos por la comunidad Yuhmu de Ixtenco, Tlaxcala, México. Scientia Fungorum 49, 1-15. https://doi.org/10.33885/sf.2019.49.1230

Novaković S, Đekic I, Klaus A, Vunduk J, Đorđević V, Tomovic V, Šojić $B$, Kocić-Tanackov S Tomašević I. 2020. Antioxidant activity of mushrooms in vitro and in Frankfurters. Meat Technology 61, 6269. https://doi.org/10.18485/meattech.2020.61.1.5

Pérez-Moreno J, Guerin-Laguette A, Arzú RF, Yu FQ, Verbeken A. 2020. Setting the scene. In: Pérez-Moreno J, Guerin-Laguette A, Arzú RF, Yu FQ (eds.), Mushrooms humans y nature in a changing world. Springer Nature, Cham. 3-28. https://doi.org/10.1007/9783-030-37378-8 1

Pumtes P, Rojsuntornkitti K, Kongbangkerd T, Jittrepotch N. 2016. Effects of different extracting conditions on antioxidant activities of Pleurotus flabellatus. International Food Research Journal 23, 173-179.

Sánchez C. 2017. Bioactives from mushroom and their application. In: Puri M (ed.), Food Bioactives. Springer, Cham. 23-57. https://doi. org/10.1007/978-3-319-51639-4 2

Sánchez JE, Liedo P. 2017. Propiedades antioxidantes de Pleurotus spp. In: Sánchez JE. Royse DJ (eds.), La biología, el cultivo y las propiedades nutricionales y medicinales de las setas Pleurotus spp. ECOSUR, San Cristobal de las Casas. 197-210.

Soares AA, De Souza CGM, Daniel FM, Ferrari GP, Da Costa SMG, Peralta RM. 2009. Antioxidant activity and total phenolic content of Agaricus brasiliensis (Agaricus blazei Murril) in two stages of maturity. Food Chemistry 112, 775-781. https://doi.org/10.1016/j. foodchem.2008.05.117

Turkoglu A, Duru ME, Mercan N, Kivrak I, Gezer K. 2007. Antioxidant and antimicrobial activities of Laetiporus sulphureus (Bull.). Murrill. Food Chemistry 101, 267-273. https://doi.org/10.1016/j.foodchem.2006.01.025

Valencia-Avilés E, Ignacio-Figueroa I, Sosa-Martínez E, Bartolomé-Camacho MC, Martínez-Flores HE, García-Pérez ME. 2017. Polifenoles: propiedades antioxidantes y toxicológicas. Revista de la Facultad de Ciencias Químicas. 16, 15-29

Valko M, Leibfritz D, Moncol J, Cronin MT, Mazur M, Telser J. 2007. Free radicals and antioxidants in normal physiological functions and human disease. The International Journal of Biochemistry and Cell Biology 39, 44-84. https://doi.org/10.1016/j.biocel.2006.07.001

Wang Y, Xu B. 2014. Distribution of antioxidant activities and total phenolic contents in acetone, ethanol, water and hot water extracts from 20 edible mushrooms via sequential extraction. Austin Journal of Nutrition and Food Sciences 2, 1-5.

Yahia EM, Gutierrez-Orozco F, Moreno-Pérez MA. 2017. Identification of phenolic compounds by liquid chromatography-mass spectrometry in seventeen species of wild mushrooms in Central Mexico and determination of their antioxidant activity and bioactive compounds. Food Chemistry 226, 14-22. https://doi.org/10.1016/j. foodchem.2017.01.044

Zamora-Martínez MC, González-Hernández A, Islas-Gutiérrez F, Cortés-Barrera EN, López-Valdez LI. 2014. Distribución geográfica y ecológica de 13 especies de hongos silvestres comestibles en Oaxaca. Revista Mexicana de Ciencias Forestales 5, 76-93. 